\title{
Development of Method for Detection of Rhodamine B Dye in Chilli Powder (Capsicum annuum L.)
}

\author{
Kirti Kapoor ${ }^{1}$, Manju Bala, Poonam Choudhary, D. Mridula, R.K. Singh
}

10.18805/ajdfr.DR-1733

\begin{abstract}
Background: Chilli is a major spice consumed all over the world. There are reports that chilli is commonly adulterated with Rhodamine $\mathrm{B}(\mathrm{Rh} \mathrm{B})$ which is a fluorescent but carcinogenic and neurogenic dye. To detect the presence of Rhodamine B dye in chilli powder, fluorescent spectroscopic method was developed.

Methods: Adulterated samples of chilli were prepared by spiking chilli powder with Rhodamine B dye at different concentrations. Effect of different solvents for extraction of dye was studied and fluorescent spectroscopy based method for quantitative detection of Rhodamine $\mathrm{B}$ dye in chilli powder was developed. Limit of detection, limit of quantification, linearity and recovery studies were carried out.

Result: Rhodamine B dye showed maximum intensity at $577 \mathrm{~nm}$ emission wavelength. Solvent effect showed that among the studied solvents; the highest and lowest fluorescence intensity of dye was observed in methanol and water, respectively. Standard curve of Rhodamine B dye was prepared in the range of $0.4-2.4 \mu \mathrm{g} / \mathrm{ml}$ concentration. Under experimental conditions, limit of detection and limit of quantification for pure dye was $0.19 \mu \mathrm{g} / \mathrm{ml}$ and $0.63 \mu \mathrm{g} / \mathrm{ml}$ and for dye in chilli matrix was $0.42 \mu \mathrm{g} / \mathrm{g}$ and $1.47 \mu \mathrm{g} / \mathrm{g}$, respectively. The developed method could detect Rhodamine B dye in spiked chilli samples with recovery limit of $93 \%$. Fluorescent spectroscopy based developed method is sensitive and capable of detecting Rhodamine B dye in microgram concentration.
\end{abstract}

Key words: Adulteration, Chilli powder, Detection method, Fluorescent spectroscopy, Rhodamine B dye.

\section{INTRODUCTION}

Chilli (Capsicum annuum L.) belonging to family Solanaceae is a major spice consumed all over the world. India is the largest producer and consumer of chilli in the world. Chilli is also known as hot pepper or bell pepper, paprika, red pepper. Chilli has sharp acidic flavor and color. Red pigment in chilli is due to presence of capsanthin and pungency is due to capsaicin content. Chilli is rich source of vitamins $(A, B$ complex and $C$ ), minerals (potassium, iron, magnesium, flavonoids) and carotenoid pigments (Maoka et al., 2001). Chilli has been reported to show various activities like antibacterial, anti-carcinogenic, analgesic, anti-diabetic etc. Chilli is consumed as such and finds enormous food applications in the powdered form.

Chilli powder is produced from the dried raw chilli. Chilli loses its color during drying and processing into powder form.In order to enhance its red color and reduce cost, many traders adulterate chilli powder illegally by the use of artificial colors,which are usually banned industrial dyes (Chen et al., 2017). Adulteration is an act of intentionally decreasing the quality of food either by mixing or substitution of inferior substances or by removal of some valuable ingredients (Bansal et al., 2017). Adulteration is reported as reliable and economical method of restoring or providing color to a processed product (Li et al., 2013). Rhodamine B dye has been reported to be used as a food additive (Jain et al., 2007) at a risk to the health of buyers (Baldev et al., 2013). Somereportsstated that Rhodamine B dye is a nonpermitted, non-food dye and used in lipsticks, rouge, nail polishes (Ozkantar et al., 2017), in street foods like coconut
Division of Food Grains and Oilseeds Processing, Central Institute of Post-harvest Engineering and Technology, Ludhiana-141 004, Punjab, India.

${ }^{1}$ University Institute of Biotechnology, Chandigarh University, Mohali-140 413, Punjab, India.

Corresponding Author: Manju Bala, Division of Food Grains and Oilseeds Processing, Central Institute of Post-harvest Engineering and Technology, Ludhiana-141 004, Punjab, India.

Email: manju.bala1@icar.gov.in

How to cite this article: Kapoor, K., Bala, M., Choudhary, P., Mridula, D. and Singh, R.K. (2022). Development of Method for Detection of Rhodamine B Dye in Chilli Powder (Capsicum annuum L.). Asian Journal of Dairy and Food Research. DOI: $10.18805 / a j d f r . D R-1733$.

Submitted: 26-05-2021 Accepted: 05-01-2022

Online: $22-02-2022$

burfi, jilebi (Meerarani et al.,1997) and in spices like chilli powder (Fu et al., 2015).

Rhodamine $\mathrm{B}$ dye $(\mathrm{RhB})$ is a synthetic and fluorescent dye, belonging to methyl-xanthene family. It is soluble in water and alcohols (ethanol and methanol). Due to its fluorescent properties, it is used as a pathological marker, dye laser and tracers for laser induced fluorescence and used in flow cytometry, ELISA, fluorescent microscopy (AIKadhemy et al., 2011; Alesso et al., 2012 and Zehentbauer et al., 2014). It has good optic properties, stability and high effectiveness (Yu et al., 2001 and Song et al., 2010). Rhodamine $B$ dye has been declared genotoxic and carcinogenic by European Food Safety Authority (EFSA). According to International Agency for Research on Cancer, 
1987 (IARC) Rhodamine B is cancer-causing and poisonous for human beings. It can cause chronic diseases by ingestion, inhalation and skin contact (Fu et al., 2015). It is harmful for gastric and intestinal tracts and showed problems like inflammation, irritation and vomiting. Rhodamine B dye is a restricted dye according to Prevention of Food Adulteration Act (PFA) in 1954 by Government of India. Safitri et al. (2015) found that when Rhodamine B dye was ingested in body through food, it increased the oxidative stress on ovarian follicles and decreased the number of primary, secondary, tertiary and graffian follicles.

Considering its illegal and unethical use in different food products, various researchers have developed different methods to detect it. Gresshma et al., 2012 developed thin layer chromatography, UV-Vis spectroscopy, paper chromatography and high-performance liquid chromatography for the detection of Rhodamine B dye in street foods. Ozkantar et al., 2017 studied the detection of dyes with different methods viz. mass spectroscopy, fluorescence spectroscopy, phosphorescence spectroscopy and UV-Vis spectroscopy. There are limited studies which focus on fluorescent spectroscopy for the detection of Rhodamine B dye in chilli. Keeping this in view, the present study was carried out to develop fluorescent spectroscopy based method for detection of Rhodamine $B$ dye in chilli powder.

\section{MATERIALS AND METHODS Reagents}

Rhodamine B standard (RhB, AR) was purchased from Sisco Research Laboratory (Mumbai, Maharashtra, India). All other chemicals used were of analytical grade. All the solutions were prepared freshly. Raw chilli and unknown samples were purchased from local market of Ludhiana. Raw chilli was pulverized in Hammer mill to obtain chilli powder at ICARCIPHET, Ludhiana during the year 2019-2020. This chilli powder was considered as pure chilli and used as reference sample. Each sample $(100 \mathrm{mg})$ was precisely weighed and transferred in a $1.5 \mathrm{ml}$ micro-centrifuge tube and extracted with $1.5 \mathrm{ml}$ of methanol twice using centrifugation at 1300 rpm for 5 minutes and supernatant was collected. Adulterated samples of chilli $(1 \mathrm{~g})$ were prepared by spiking chilli powder with solution of Rhodamine $B$ dye at different concentrations ranged from $0.8-4.8 \mu \mathrm{g} / \mathrm{ml}$ and mixed to obtain uniform sample. Each spiked sample was extracted according to the above steps.

Fluorescent spectrophotometer (Varian Cary eclipseFL1004M013) equipped with Xenon flash lamp was used for recording flouremetric measurements. Pure chilli and Rhodamine $B$ dye were scanned to get their intensity peaks at specific wavelengths using fluorescent spectrophotometer in the range of $200-1100 \mathrm{~nm}$. Rhodamine $B$ dye is a fluorescent dye so it get excited and emit light at specific wavelength.

To confirm the accuracy of this method, limit of detection (LOD), limit of quantification (LOQ) and recovery studies were performed. The emission wavelength for Rhodamine B dye was set at $577 \mathrm{~nm}$. Six linear concentrations $(0.4,0.8$,
$1.2,1.6,2.0,2.4 \mu \mathrm{g} / \mathrm{ml}$ ) of working standards of pure Rhodamine $B$ dye and $0.8-4.8 \mu \mathrm{g} / \mathrm{ml}$ concentrations of Rhodamine B dye spiked in chilli matrix $(1 \mathrm{~g})$ were used in three replications and regression curve were drawn. LOD and $\mathrm{LOQ}$ were determined from these curves. The developed method for estimation of residues of Rhodamine $B$ dye in chilli powder was validated by conducting recovery studies using chilli samples. Per cent recovery was calculated by using following formula:

Per cent recovery $=$

Quantity of Rhodamine B dye recovered

$$
\text { Quantity of Rhodamine B dye added } \times 100
$$

\section{RESULTS AND DISCUSSION}

\section{Selection of solvent}

Rhodamine B dye in aqueous solution exists in different forms such as ionized species, neutral form, lactone and molecular aggregates, which are characterized by typical absorption and fluorescent spectra, depending on solvent and other factors. Selection of solvent affects the fluorescence intensity of dye as well as its extraction from the matrix. In order to find the best solvent, Rhodamine $B$ dye was dissolved in different organic solvents and its emission spectra was obtained as shown in Fig 1(A) and Fig 1(B). Different solvents viz. water, methanol, ethanol, methanol in acetonitrile and DMSO (Di-methyl sulfoxide) were studied. The results showed that the solvents influenced the

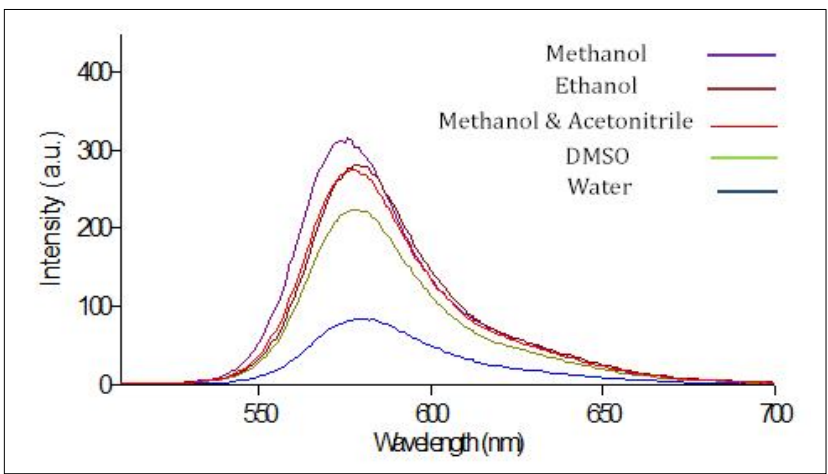

Fig 1(A): Effect of different solvents on intensity of Rhodamine B dye using fluorescent spectrophotometer (2D).

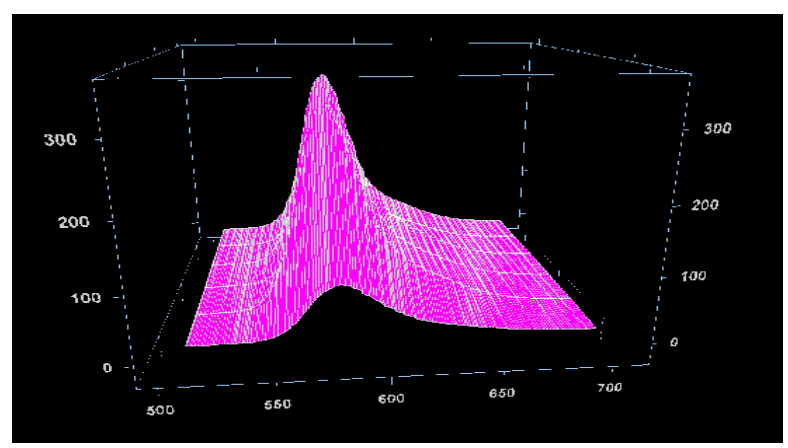

Fig 1(B): Effect of different solvents on intensity of Rhodamine B dye using fluorescent spectrophotometer (3D). 
fluorescent intensity and extraction of Rhodamine B dye differently (Table 1). The spectra obtained with different solvents differed in terms of peak wavelength and intensity. However, the spectral shapes were virtually identical. The shortest peak wavelength and the highest intensity were observed for methanol while the longest peak wavelength and the lowest intensity were observed for water among the studied solvents. Low intensity indicates less extraction in water which could be due to the fact that water showed poor permeability for dye as compared to other solvents. Deviations in spectral intensity could be due to variable solvation of solvents. Our results are in accordance with results of Zehentbauer et al. (2014) and Fu et al. (2015) who have reported effect of solvents on emission characteristic of dye and found methanol as the best solvent. Considering good extraction and good fluorescence properties of Rhodamine $B$ dye in methanol, it was used as solvent for further studies.

\section{Spectral properties of Rhodamine B dye}

Rhodamine B dyes and pure chilli powder were dissolved in methanol and scanned to get emission wavelength and intensity using fluorescent spectrophotometer. Pure chilli powder in solution did not show any peak in the whole wavelength range. Pure dye in solution gave maximum intensity at $577 \mathrm{~nm}$ emission wavelength as shown in Fig 2 and excitation wavelength was observed at $555 \mathrm{~nm}$. Similar

Table 1: Effect of different solvents on the intensity of Rhodamine B dye.

\begin{tabular}{lc}
\hline Solvent & Intensity \\
\hline Water & 84.12 \\
Methanol & 315.9 \\
Ethanol & 280 \\
Methanol : Acetonitrile (1:1) & 277 \\
DMSO & 218 \\
\hline
\end{tabular}

Table 2: Intensity of different concentrations of Pure Rhodamine B dye in methanol.

\begin{tabular}{lr}
\hline Conc. of Rhodamine B dye $(\mu \mathrm{g} / \mathrm{ml})$ & Intensity \\
\hline 0.4 & 51.74 \\
0.8 & 101.82 \\
1.2 & 150.68 \\
1.6 & 199.59 \\
2.0 & 252.49 \\
\hline
\end{tabular}

Table 3: Intensity of different concentrations of Rhodamine B dye in chilli matrix.

\begin{tabular}{lcc}
\hline Sr. no & $\begin{array}{c}\text { Concentration of } \\
\text { Rhodamine B dye }(\mu \mathrm{g} / \mathrm{g})\end{array}$ & Intensity \\
\hline 1. & 0.8 & 74.59 \\
2. & 1.6 & 155.39 \\
3. & 2.4 & 202.77 \\
4. & 3.2 & 286.54 \\
5. & 4.0 & 325.82 \\
6. & 4.8 & 389.98 \\
Pure Rhodamine & - & 551.69 \\
Pure Chilli & - & - \\
\hline
\end{tabular}

spectral properties of Rhodamine B dye in solution using fluorescent spectrophotometer have been reported with maximum intensity at $580 \mathrm{~nm}$ and $576 \mathrm{~nm}$ emission wavelengths, respectively (Al-Kadhemy et al., 2011 and Alesso et al., 2012). While using synchronous fluorescence approach, Fu et al. (2015) observed maximum intensity at $570 \mathrm{~nm}$ wavelength for Rhodamine B dye pure solution.

\section{Standard curve of Rhodamine B dye}

For quantitative analysis of Rhodamine B dye, standard solutions of pure dye were prepared in concentrations range of $0.4-2.0 \mu \mathrm{g} / \mathrm{ml}$ as shown in Table 2 and as concentration of Rhodamine $B$ dye was increasing, the peak and wavelength were increasing as shown in Fig 3(A) and Fig 3(B). Similarly, Rhodamine B standards were spiked in the increasing concentration into the chilli powder, the intensities of chilli powder extract were obtained as shown in Table 3.

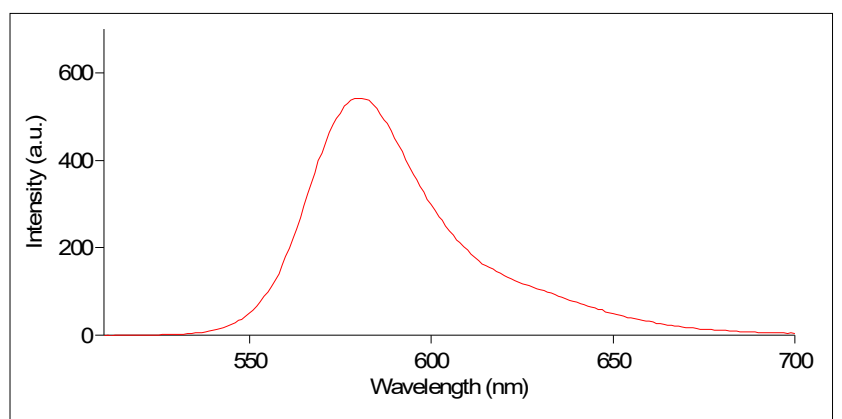

Fig 2: Emission spectra of Rhodamine B dye in methanol using fluorescent spectrophotometer.

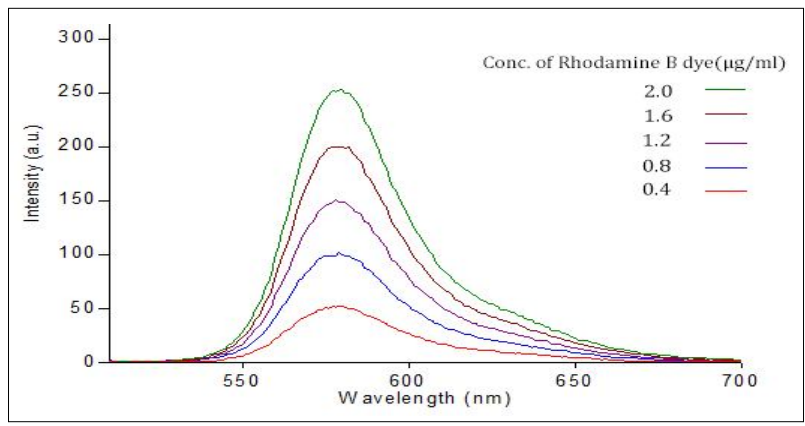

Fig 3(A): Emission spectra of different concentrations of pure Rhodamine B dye using fluorescent spectrophotometer (2D).

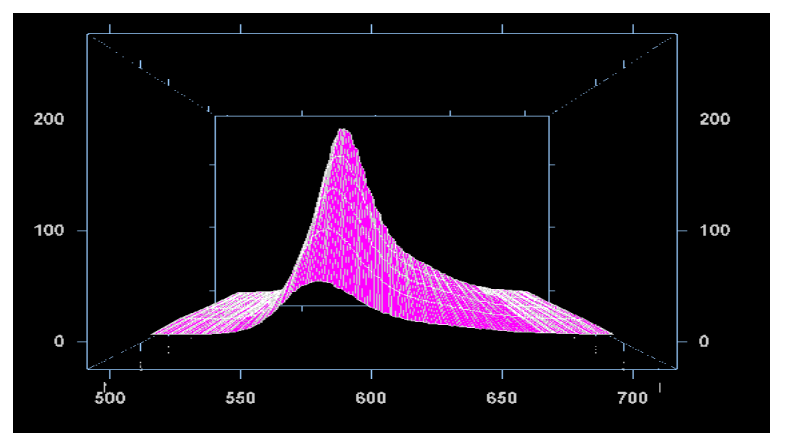

Fig 3(B): Emission spectra of different concentrations of pure Rhodamine B dye using fluorescent spectrophotometer (3D). 
The chilli powder extract alone did not give any peak. With increasing concentration of rhodamine B fluorescence signal increased gradually. The peaks of Rhodamine B in chilli powder extract shifted towards longer wavelength as shown in Fig 4(A) and Fig 4(B). This phenomenon could be due to the interference by the color of chilli powder extract. Fu et al., 2015 has reported that bright red and complex matrix of chilli powder largely influence the response of the Rhodamine B. In order to remove the matrix effects, continuous standard addition method was used. Rhodamine $B$ concentration was calculated by spiking known concentration of Rhodamine B standard solution into the chilli sample. Similarly, Al-Kadhemy et al. (2011) has also reported the effect of concentration of Rhodamine $B$ dye in ethanol on fluorescent spectrum using different concentrations $1 \times 10^{-2}, 1 \times 10^{-3}, 1 \times 10^{-4}$ and $1 \times 10^{-5} \mathrm{~mol} / \mathrm{liter}$.

Table 4: Recovery of Rhodamine B dye from spiked chilli samples.

\begin{tabular}{lcr}
\hline $\begin{array}{l}\text { Conc. of Rhodamine } \\
\text { B dye }(\mu \mathrm{g} / \mathrm{g})\end{array}$ & $\begin{array}{c}\text { Detected } \\
\text { amount }(\mu \mathrm{g} / \mathrm{g})\end{array}$ & Recovery $(\%)$ \\
\hline 1.6 & 1.46 & 91.25 \\
2.4 & 2.23 & 92.91 \\
3.2 & 2.93 & 91.56 \\
3.6 & 3.23 & 89.72 \\
\hline
\end{tabular}

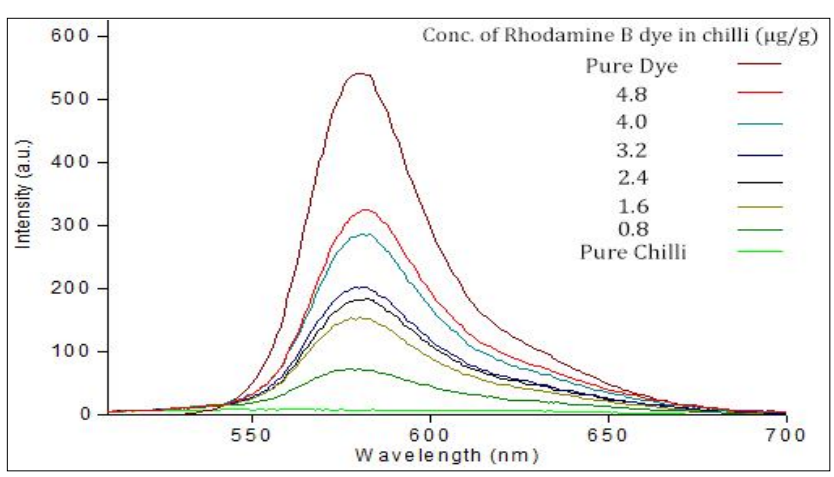

Fig 4(A): Emission spectra of different concentrations of pure Rhodamine $\mathrm{B}$ dye in chilli matrix using fluorescent spectrophotometer (2D).

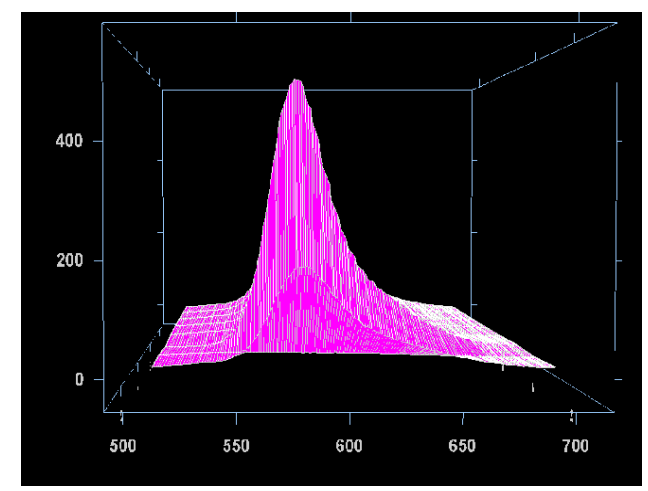

Fig 4(B): Emission spectra of different concentrations of pure Rhodamine $B$ dye in chilli matrix using fluorescent spectrophotometer (3D).

\section{Analytical performance}

Under the optimized conditions Rhodamine B could be analyzed by the proposed method. Various characteristics such as limit of detection, limit of quantification and recoveries were calculated for the developed method using regression equation. Various amounts of Rhodamine B standard solutions were prepared in methanol to prepare a series of working solutions. L.O.D for pure Rhodamine B dye was 0.19 $\mu \mathrm{g} / \mathrm{ml}$ and L.O.Q. was $0.63 \mu \mathrm{g} / \mathrm{ml}$. Similarly, various amounts of Rhodamine B standard solutions were spiked into the blank extract to prepare a series of working solution coexisting with the chilli matrix. L.O.D and L.O.Q of Rhodamine B dye in chilli matrix was $0.42 \mu \mathrm{g} / \mathrm{g}$ and $1.47 \mu \mathrm{g} / \mathrm{g}$. Correspondingly, Alesso et al. (2012) showed analytical results for limit of detection and limit of quantification to determine the Rhodamine B dye such as $1.40 \times 10^{-2} \mu \mathrm{g} / \mathrm{l}$ and $4.67 \times 10^{-2} \mu \mathrm{g} / \mathrm{l}$. Fu et al. (2015) reported limit of detection for Rhodamine B dye in chilli powder was $1.3 \mathrm{ng} / \mathrm{g}$.

To validate the accuracy of above method, recovery experiments were performed. The recovery of Rhodamine $B$ dye was found by standard addition method. Chilli sample was spiked by different amounts of Rhodamine B standard solution as shown in Table 4. The data revealed that the spiked and detected amounts of Rhodamine B were approximately identical. Recovery percentage of Rhodamine $B$ dye in chilli powder was $89.72-92.91 \%$. Variable recoveries of Rhodamine B have been reported by other researchers in the range of $90-100 \%$ from different chilli products (chilli oil, chilli powder and chilli sauce) by Fu et al. (2015) while $95-100 \%$ has been reported in different condiments by Alesso et al. (2012).

\section{CONCLUSION}

In the present study, a method was developed to detect Rhodamine B dye in chilli powder using fluorescent spectroscopy. The developed method is a simple, reliable and sensitive for determination of Rhodamine $B$ dye in chilli powder. Scan studies showed emission maxima of Rhodamine B dye at $577 \mathrm{~nm}$ and excitation at $555 \mathrm{~nm}$. The limit of detection and limit of quantification for pure dyewas $0.19 \mu \mathrm{g} / \mathrm{ml}$ and $0.63 \mu \mathrm{g} / \mathrm{ml}$ whereas for Rhodamine $B$ dye in chilli powder was $0.42 \mu \mathrm{g} / \mathrm{g}$ and $1.47 \mu \mathrm{g} / \mathrm{g}$, respectively. Among the studied solvents, methanol showed best results in terms of emission intensity of Rhodamine B dye. About 90 to $93 \%$ recovery of Rhodamine B dye was observed. Proposed methodology is useful for Rhodamine $B$ dye estimation in chilli powder.

Conflict of interest: None.

\section{REFERENCES}

Alesso, M., Bondioli, G., Talío, M.C., Luconi, M.O. and Fernández, L.P. (2012). Micelles mediated separation fluorimetric methodology for Rhodamine $B$ determination in condiments, snacks and candies. Food Chemistry. 134(1): 513-517. 
Al-Kadhemy, M.F., Alsharuee, I.F. and Al-Zuky, A.A.D. (2011). Analysis of the effect of the concentration of rhodamine $B$ in ethanol on the fluorescence spectrum using the 'Gauss Mod' function. Journal of Physical Science. 22(2): 77-86.

Baldev, E., Mubarak Ali, D., Ilavarasi, A., Pandiaraj, D., Ishack, K.S.S. and Thajuddin, N. (2013). Degradation of synthetic dye, Rhodamine $B$ to environmentally non-toxic products using microalgae. Colloids and Surfaces B: Biointerfaces. 105, 207-214.

Bansal, S., Singh, A., Mangal, M., Mangal, A.K. and Kumar, S. (2017). Food adulteration: Sources, health risks and detection methods. Critical Reviews in Food Science and Nutrition. 57(6): 1174-1189.

Chen, D.Y., Zhang, H., Feng, J.L., Zeng, D., Ding, L., Liu, X.J. and $\mathrm{Li}, \mathrm{B} . \mathrm{R}$. (2017). Research on the determination of 10 industrial dyes in food stuffs. Journal of Chromatographic Science. 55(10): 1021-1025.

Fu, D.S., Wu, P.P., Zhong, X.D., Liu, Q., Luo, H.D. and Li, Y.Q. (2015). A simple synchronous fluorescence approach for rapid and sensitive determination of Rhodamine $B$ in chilli products. Food Analytical Methods. 8(1): 189-194.

Gresshma, R.L. and Paul, M.R. (2012). Qualitative and quantitative detection of Rhodamine $B$ extracted from different food items using visible spectrophotometry. Malaysian Journal of Forensic Sciences. 3(1): 36-40.

International Agency for Research on Cancer (IARC) (1987). Overall evaluations of carcinogenicity: An updating of IARC Monographs volumes 1 to 42 . Lyon, France: IARC. (p. 53).

Jain, R., Mathur, M., Sikarwar, S. and Mittal, A. (2007). Removal of the hazardous dye rhodamine $B$ through photocatalytic and adsorption treatments. Journal of Environmental Management. 85(4): 956-964.
Li, J., Ding, X.M., Liu, D.D., Guo, F., Chen, Y., Zhang, Y. B. and Liu, H. M. (2013). Simultaneous determination of eight illegal dyes in chilli products by liquid chromatographytandem mass spectrometry. Journal of Chromatography B. $942: 46-52$.

Maoka, T., Mochida, K., Kozuka, M., Y., Fujiwara, Y., Hashimoto, K. And Nishino, H. (2001). Cancer chemopreventive activity of carotenoids in the fruits of Red Paprika Capsicum Annuum L. Cancer letters. 172(2): 103-109.

Meerarani, S., Ramadass, P., Padmanaban, V.D. and Nachimuthu, K. (1997). Incidence of aflatoxin M1 in milk samples around Chennai (Madras) city. Journal of Food Science and Technology. 34(6): 506-508.

Özkantar, N., Soylak, M. and Tuzen M. (2017). Spectrophotometric detection of rhodamine $B$ in tap water, lipstick, rouge and nail polish samples after supramolecular solvent microextraction. Turkish Journal of Chemistry. 41(6): 987-994.

Safitri, Y.A., Indrawan, I.W.A. and Winarsih, S. (2015). Rhodamine $B$ induces oxidative stress and cervical epithelial cell proliferation in the uterus. Toxicology reports. 2: 1434-1436.

Song, S., Lin, F., Liu, L., Kuang, H., Wang, L. and Xu, C. (2010). Immunoaffinity removal and immunoassay for Rhodamine B in chilli powder. Journal of Food Science and Technology. 45(12): 2589-2595.

Yu, J.S. and Zhou, T.Y. (2001). The electrochemistry and thin-layer luminescence spectroelectrochemistry of Rhodamine $6 \mathrm{G}$ at a 4, 42-bipyridine-modified gold electrode. Journal of Electro-analytical Chemistry. 504(1): 89-95.

Zehentbauer, F. M., Moretto, C., Stephen, R., Thevar, T., Gilchrist, J.R., Pokrajac, D and Kiefer, J. (2014). Fluorescence spectroscopy of Rhodamine 6G: concentration and solvent effects. Spectrochimica Acta Part A: Molecular and Biomolecular Spectroscopy. 121: 147-151. 